\title{
Why are you calling me? How study introductions change response patterns
}

\author{
Dylan M. Smith ${ }^{1,2}$, Norbert Schwarz ${ }^{2}$, Todd R. Roberts ${ }^{2} \&$ Peter A. Ubel $^{1,2}$ \\ ${ }^{1} V A$ Health Services Research and Development Center of Excellence, VA Ann Arbor Health Care System, \\ Ann Arbor, MI, USA; ${ }^{2}$ University of Michigan, Ann Arbor, MI, USA (E-mail: dylsmith@umich.edu)
}

Accepted in revised form 27 October 2005

\begin{abstract}
Purpose: Research on survey methodology has demonstrated that seemingly innocuous aspects of a survey's design, such as the order of questions, can produce biased results. The current investigation extends this work by testing whether standard survey introductions alter the observed associations between variables. Methods: In two experimental studies, we invited Parkinson's disease (PD) patients to participate in a telephone survey of (a) Parkinson's patients, conducted by a regional medical center, or (b) the general population, conducted by a regional university. The survey in Study $1(n=156)$ first assessed life-satisfaction, and subsequently health satisfaction. In Study $2(\mathrm{n}=99)$, we reversed the order of the two questions, asking the health questions first. Results: When the introduction focused on Parkinson's disease, we observed an increased correlation between life-satisfaction and a later question about health satisfaction ( $r=0.34$ vs. 0.63 after general population versus Parkinson's introduction, respectively; Study 1). In Study 2 , asking the health questions first resulted in high correlations regardless of the introduction; in addition, judgments of life-satisfaction were lower after the Parkinson's-focused introduction. Conclusions: When participants were informed prior to the survey that its purpose was to examine well-being in PD, health satisfaction was a much more important component of life-satisfaction, accounting for three times as much variation. We hypothesize that the survey introduction primed participants' health status, resulting in an artificially large correlation with life-satisfaction.
\end{abstract}

Key words: survey methodology, context effects

\section{Introduction}

Preceding questions can profoundly influence respondents' answers to subsequent ones (for reviews see Refs. [1, 2]). The present studies extend the theoretical rationale developed for understanding question order effects to research introductions. In survey research, introductions commonly provide respondents with general information about the purpose of the study, the affiliation of the researcher, and the study's sponsor to meet informed consent requirements. We propose that these introductions increase the accessibility of related information and hence the likelihood that this information is considered during the subsequent interview. Accordingly, introductions may influence responses in ways that parallel the operation of question order effects.

We test this hypothesis in two experiments, conducted as telephone surveys, with samples of Parkinson's disease patients living in Pennsylvania. The crucial manipulation is the interviewer's introductory statement, pertaining to the purpose of the study and the affiliation of the researchers. One group was told that the survey is "being conducted by researchers at the University of Pennsylvania Movement Disorders Clinic, and is an attempt to better understand the quality of life of people who have Parkinson's disease." The other group was told that the survey is "being 
conducted by researchers at the University of Pennsylvania and is an attempt to better understand the quality of life of people who live in the eastern United States." Of interest is the influence of these introductions on subsequent reports of general life-satisfaction and health satisfaction, as well as the relationship between these variables.

We first introduce the theoretical rationale and present the relevant data. Subsequently, we address the methodological and theoretical implications.

\section{Theoretical rationale}

When asked an attitude question, respondents can rarely retrieve a 'ready-for-use' answer from memory. Instead, they need to compute an answer on the spot, drawing on information that is accessible at that point in time. Which information respondents consider is, in part, a function of what was brought to mind by preceding questions (for reviews see Refs. [1-3]). This affects measures of association as well as mean differences.

\section{Associations between variables}

Strack et al. [4] asked students how satisfied they are with their lives in general and how often they have a date. When the life-satisfaction question preceded the dating frequency question, the answers correlated $r=-0.12$; but when the question order was reversed, this correlation increased to $r=0.66$. Such findings reflect that respondents can draw on many different aspects of their lives to arrive at a general satisfaction judgment (for a review see Ref. [5]). Which aspects they consider depends on what is most likely to come to mind (for other examples see Refs. [6-8]). Importantly, such accessibility effects can profoundly affect the substantive conclusions drawn. In the above example, dating frequency accounts for either a negligible $1.4 \%$ or an impressive $43.6 \%$ of the variance in life-satisfaction [4], depending on the order in which the questions were asked.

We propose that the same logic holds for survey introductions. When respondents suffering from Parkinson's disease are told that the study investigates the well-being of Parkinson's patients, health-related aspects of their lives are more likely to come to mind than when they are told that the survey investigates the well-being of 'people living in the eastern United States.' These health-related aspects are likely to be included in the mental representation that respondents form of their livesas-a-whole, resulting in assimilation effects [9].

In fact, there are good theoretical reasons to assume that the influence of introductions may be even more pronounced than the influence of preceding questions, in particular when the purpose of the study is reinforced by the researcher's affiliation. In general, respondents try to be cooperative informants and attempt to provide information that is useful to the researcher. To do so, they take the researcher's perceived epistemic interest into account in an attempt to make their answers as informative as possible (for a review see Ref. [10]). For example, Norenzayan and Schwarz [11] provided students with a newspaper story about a postal worker who killed his former colleagues after he lost his job and asked them to explain, in a free response format, why the event happened. To manipulate respondents' assumptions about the researcher's likely epistemic interest, the questionnaire was printed either on the letterhead of an 'Institute for Personality Research' or the letterhead of an 'Institute for Social Research.' As expected, respondents' took the researcher's likely epistemic interest into account and focused on the variables they could assume to be most relevant to the researcher's goals. Hence, they provided more personality-based explanations when the letterhead suggested the researcher is a personality psychologist, but more situational explanations when it suggested the researcher is a social scientist. Accordingly, providing the 'University of Pennsylvania Movement Disorders Clinic,' rather than the more generic "University of Pennsylvania," as the researcher's affiliation may further contribute to respondents' consideration of health-related aspects of their lives.

If a Parkinson's-focused introduction increases the extent to which respondents consider healthrelated aspects of their lives, these aspects should figure more prominently in their general life-satisfaction judgments. We should therefore observe a higher correlation between an initial judgment of general life-satisfaction and a subsequent health question when the survey is introduced as a survey of Parkinson's patients conducted by a University of Pennsylvania medical center rather than as survey of 'people living in the eastern United 
States' conducted by the University of Pennsylvania. Study 1 tests this hypothesis.

\section{Mean differences}

The influence of preceding questions can also be observed in mean differences, provided that the analyses take the implications of the accessible information into account. For example, Schwarz et al. [8] observed that a preceding question about marital satisfaction increased reported general lifesatisfaction for happily married respondents, but decreased it for unhappily married respondents. We expect that introductions can affect mean differences in the same way. If so, we should observe that respondents who are relatively satisfied with their health report higher, and respondents who are relatively dissatisfied lower, general life-satisfaction when the survey introduction focuses their attention on health-related information.

However, a potential complication deserves attention. When the study is introduced as a general population survey, the relevant frame of reference for rating one's own life-satisfaction is the general population. On the other hand, when the study is introduced as a survey of Parkinson's patients, the relevant frame of reference is other Parkinson's patients. Moreover, under the latter introduction, the interviewer and researcher are obviously aware that the respondent is a Parkinson's patient, or else the respondent would not have been called. This makes the respondent's health status part of the common ground of the conversation and the respondent's answers may be designed with this background in mind (for a conceptual discussion see Ref. [10, 12]). If so, the Parkinson's-focused introduction may result in a general upward shift in satisfaction judgments relative to the general population introduction, reflecting that respondents apply different frames of reference or scale anchors in making their ratings. Study 1 allows us to assess these possibilities.

\section{Study 1: General-specific question order}

Parkinson's patients living in Pennsylvania participated in an experiment introduced either as a survey of Parkinson's patients, conducted by a University of Pennsylvania medical center or as a survey of residents of the eastern United States, conducted by the University of Pennsylvania. All respondents reported first on their general lifesatisfaction and next on their health satisfaction.

\section{Method}

Note that the purpose of this study is to test specific hypotheses about survey introductions in an experimental design with random assignment to conditions, not to conduct a representative survey of Parkinson's patients. Our procedures reflect this goal. First, we recruited Parkinson's patients from a clinical data base, as described below, using criteria that rendered it likely that a sufficient number of participants could be obtained within budget constraints. Second, when respondents answered the phone, they were asked to participate in a survey on happiness and life-satisfaction conducted by the University of Pennsylvania. Once they agreed to participate, the computer assisted telephone interview (CATI) system assigned them to one of two experimental conditions and they received more detailed information about the purpose of the study and the specific sponsor, as described below. This procedure ensured that the respondents as well as the interviewers were blind to conditions at the recruitment stage, thus avoiding differential response rates as a function of survey introduction.

\section{Participants}

Respondents for this experiment were drawn from the University of Pennsylvania Health System's (UPHS) computerized database of 770,000 patients from the Delaware Valley area. Our recruitment procedures were designed to maximize the feasibility of the study within budget constraints. First, we increased the chance of having accurate contact information by selecting patients with a diagnosis of Parkinson's disease who had visited the neurology clinic at least three times in the prior two years. Second, we sorted the call list so that people who had visited the clinic most recently would be called first. Third, to increase the odds of contacting patients who were mentally competent and able to answer a phone survey, we first contacted patients who were less than 70 years old, followed by patients $70-80$ years old. Fourth, 
patients who had recently been enrolled in another quality of life study being conducted concurrently at the University of Pennsylvania $(n=180)$ were dropped from the list. Finally, once contacted, respondents were offered an incentive of $\$ 5$ for their participation.

A total of 849 phone numbers were called, and 469 Parkinson's patients were actually contacted. Of these, 156 completed the interview. Among the 313 who were contacted but did not complete the interview, 171 refused to participate, 138 requested a callback but were not contacted again, and 4 did not speak English. Three of the respondents who completed the interview declined to provide consent for the use of their information at the conclusion of the interview, and two failed to respond to one of our key variables of interest, leaving 151 respondents' data available for analysis. About $45 \%$ of the final participants were female. Average age of the participants was 66 years.

\section{Procedure}

The short (less than $10 \mathrm{~min}$ ) CATI survey was conducted by MSInteractive, a marketing and research company with experience in experimental scientific research. Ten professional interviewers were used. The interviewers said that they were calling on behalf of the University of Pennsylvania to conduct a brief survey about quality of life and happiness. At this stage, interviewers were not aware of the respondent's subsequent assignment to experimental conditions, thus precluding self-selection and differential response rates.

After respondents agreed to participate, the CATI system randomly assigned them to one of two introduction conditions. In the Parkinson's condition, respondents $(n=78)$ were told that the study was "being conducted by researchers at the University of Pennsylvania Movement Disorders Clinic, and is an attempt to better understand the quality of life people who have Parkinson's disease." In the general public condition, respondents $(n=73)$ were told that the survey was "being conducted by researchers at the University of Pennsylvania and is an attempt to better understand the quality of life of people who live in the eastern United States."
Following these introductions, respondents were asked, "How satisfied are you with your life as a whole these days?", and next, "How satisfied are you with your current health?" They answered both questions by selecting a value between 1 ("completely dissatisfied") and 10 ("completely satisfied"). Additional questions addressed topics unrelated to the present analyses. Because the data were collected in telephone interviews, respondents were not aware of the content of later questions when they answered an earlier one.

\section{Debriefing and consent}

At the conclusion of the interview, respondents assigned to the general public condition were informed that the study was targeted to Parkinson's patients and that they had been recruited for this reason. They were given the option of having their data excluded from the study if they felt they would not have given consent to participate had they known the selection criterion. Two participants exercised this option and their data were destroyed.

The data were kept strictly confidential and all contact information was destroyed after data collection and participant payment.

\section{Results and discussion}

We first address how the different introductions affect the relationship between variables and subsequently turn to mean differences.

\section{Measures of association}

When the study was introduced as a general population survey of well-being conducted by the University of Pennsylvania, respondents' reports of general life-satisfaction correlated $r=0.34$ $(n=73)$ with their subsequent reports of health-satisfaction. ${ }^{1}$ As expected, these correlations increased significantly when the study was introduced as a survey of the well-being of Parkinson's patients conducted by a medical center. In this case, respondents' reports of general lifesatisfaction correlated $r=0.63(\mathrm{n}=78)$ with their subsequent reports of health-satisfaction. See Table 1. 
Table 1. Correlations ( $r$ 's) between Life Satisfaction and Health Satisfaction as a function of survey introduction and question order

\begin{tabular}{lllll}
\hline Study & Introduction & Question order & $r$ & $\mathrm{~N}$ \\
\hline 1 & Parkinsons & Life/health & 0.63 & 78 \\
1 & General public & Life/health & 0.34 & 73 \\
2 & Parkinsons & Health/life & 0.47 & 50 \\
2 & General public & Health/life & 0.56 & 48 \\
\hline
\end{tabular}

All correlations are significant at $p<0.01$.

A linear regression analysis with experimental condition, health satisfaction, and the interaction term as predictors of life-satisfaction confirmed the reliability of the observed effect. The model is significant overall $(F(3,147)=17.31, \quad p<0.001)$, and a significant interaction term (regression coefficient $=-0.30$, standard error $=0.12, p<0.02$ ) indicates that the subsequent question about health satisfaction was a better predictor of general life-satisfaction when the study introduction focused on Parkinson's disease, as reflected in the differential correlations. ${ }^{2}$

\section{Mean differences}

To address mean differences, we tested the influence of the introductions on reports of health and life-satisfaction with a repeated-measures ANO$\mathrm{VA}$, treating the two satisfaction judgments as a within-subjects factor, and experimental condition (Parkinson's versus general public) as a between subjects factor. Neither the overall life-satisfaction nor the overall health-satisfaction means were significantly affected by the survey introductions. Thus, the mean ratings provide no support for the predicted upward shift in ratings as a function of changes in respondents' frame of reference. See Table 2.
However, an analysis of general life-satisfaction that takes respondents' health satisfaction into account replicates the previously observed [8] accessibility effect. We split health satisfaction at the median $(\mathrm{M}=6.0)$, and performed a 2 (high vs. low health satisfaction) $\times 2$ (Parkinsons vs. general public survey introduction) ANOVA, where we observed a significiant interaction, $F(1,147)=4.84, p<0.03$. Specifically, participants with low health satisfaction reported lower general life-satisfaction when the survey introduction brought Parkinson's disease to mind $(\mathrm{M}=5.77)$ than when it did not $(\mathrm{M}=6.65), F(1,147)=4.17$, $p<0.05$, Cohen's $d=0.40$ for the simple main effect. In contrast, participants with high healthsatisfaction reported non-significantly higher general life-satisfaction following the Parkinson's disease introduction (M's $=8.06$ vs. 7.56, n.s.). These diverging effects cancel one another, resulting in the observed absence of an overall effect of survey introduction on mean reported life-satisfaction (again paralleling the effects of question order; [8]).

\section{Discussion}

In sum, the present results replicate the patterns previously observed for question order effects. In the earlier research, questions about dating frequency [4] or marital satisfaction [8] increased respondents' consideration of these aspects of their lives in later judgments of general life-satisfaction, as indicated by increased correlations. In much the same way, introducing the study as a health survey increased respondents' consideration of health-related aspects of their lives, rendering them more influential when respondents evaluated their livesas-a-whole - even though no health-related question was asked prior to the life-satisfaction question. As a result, we would arrive at different

Table 2. Means and standard deviations of Life and Health Satisfaction as a function of survey introduction

\begin{tabular}{llll}
\hline & Parkinson's introduction & General public introduction & $p$ level \\
Study 1 (Life Sat. asked first) & & & $n . s$. \\
\hline Life satisfaction & $6.79(2.07)$ & $7.10(2.14)$ & $n . s$. \\
$\quad$ Health satisfaction & $5.96(2.26)$ & $5.75(2.58)$ & $<0.05$ \\
Study 2 (Health Sat. asked first) & & & $<.44(2.17)$ \\
$\quad$ Life satisfaction & $6.58(1.97)$ & $5.31(2.44)$ & $<0.08$ \\
Health satisfaction & $6.12(2.05)$ & & \\
\hline
\end{tabular}


conclusions about the impact of Parkinson's disease on patients' well-being depending on the introduction used. When the introduction drew attention to the disease, respondents' health satisfaction accounted for $39.7 \%$ of the variance in reported life-satisfaction; yet under a generic introduction this estimate dropped by two thirds to $11.5 \%$. Moreover, respondents who were dissatisfied with their health reported lower general life-satisfaction when the introduction drew attention to their disease, again paralleling the influence of question order effects.

\section{Study 2: Specific-general question order}

Study 2 extends our exploration of introductions by assessing their influence when the first question asked pertains to respondents' health satisfaction. We replicated the procedures of Study 1 with respondents drawn from the same pool, except that respondents' health satisfaction was assessed prior to their general life-satisfaction.

\section{Association between variables}

According to our conceptual rationale, introducing the study as a survey of Parkinson's patients brings health-related information to mind, much as a preceding health question would. If so, the observed effects of survey introduction on reports of general life-satisfaction should be attenuated or eliminated when a health satisfaction question precedes the life-satisfaction question, thus ensuring that health-related information is highly accessible independent of survey introduction. This effect should also be reflected in comparisons across both studies. Specifically, the correlations obtained for the health-life order (Study 2) under either introduction should not differ significantly from the correlation obtained for the life-health order (Study 1) when the "Parkinson's" introduction brought health concerns to mind.

\section{Mean differences}

Recall that we expected that the different survey introductions would bring different frames of reference to mind, such that respondents evaluate their lives relative to other Parkinson's patients or relative to the general population, depending on the introduction used. Study 1 provided no support for this prediction. It is conceivable that this theoretically plausible effect is limited to questions that pertain directly to respondents' health. If so, we may observe a shift in respondents' frame of reference on their health satisfaction judgments when this question is asked first, as is the case in Study 2 .

It is possible that life-satisfaction judgments will show a parallel effect due to the shift in frame of reference. However, the specific-general (i.e., health-life) question order used in Study 2 may give rise to part-whole contrast effects [13]. As Schwarz et al. [8] demonstrated, part-whole contrast effects are based on a conversational norm of nonredundancy. Speakers are expected to provide new information rather than to reiterate information that the recipient already has $[14,15]$. Hence, having just reported that their health is poor, they may interpret the subsequent question about their life in general as if it where worded, "Aside from your health, which you just told me about, how satisfied are you with other aspects of your life?" If so, we should observe higher health-satisfaction but lower life-satisfaction when the survey is introduced as a survey of Parkinson's patients rather than the general population. Study 2 bears on these possibilities.

\section{Method}

Study 2 followed the procedures of Study 1, described above, except that the data were collected several weeks later and the health satisfaction question preceded the general life-satisfaction question. The same pool of potential patients was used, although patients who participated in Study 1 were not eligible for participation in Study 2 .

\section{Participants}

A total of 389 phone numbers were called, and 261 Parkinson's patients were actually contacted. Of these, 100 completed the interview. Among the 161 who were contacted but did not complete the interview, 116 refused to participate, 43 requested a callback but were not contacted again, and 2 did not speak English. One of the respondents who 
completed the interview declined to provide consent for the use of their information at the conclusion of the interview, and one failed to answer one of our focal questions, leaving 98 respondents' data available for analysis. About $33 \%$ of the final participants were female. Average age of the participants was 64 years. Fifty participants were assigned to the Parkinsons' condition, while 48 were assigned to the general public condition.

\section{Results and discussion}

\section{Associations between variables}

Respondents' reported health satisfaction correlated with their subsequently reported general life-satisfaction $r=0.56(\mathrm{n}=48)$ when the study was introduced as a general population survey and $r=0.47(\mathrm{n}=50)$ when it was introduced as a survey of Parkinson's patients. Both correlations are not significantly different from one another, indicating that the introduction exerted no additional influence once health-related information was brought to mind by the initial question. Moreover, neither of these correlations differs significantly from the correlation observed under the 'Parkinson' introduction of Study 1, $r=0.63$, which similarly brought health-related concerns to mind (the $z$ tests for differences between two correlations in different samples revealed that both $z$ values were $<1$, n.s.). These observations are consistent with the assumption that a health-related introduction is functionally equivalent to health-related preceding questions. See Table 1.

\section{Mean differences}

We again tested the influence of survey introduction on reports of health and life-satisfaction with a repeated measures ANOVA, treating the two satisfaction judgments as a within-subjects factor, and experimental condition (Parkinson's vs. general public introduction) as a between-subjects factor. The results show a significant interaction between survey introduction and type of satisfaction judgment, $F(1,96)=15.03, p<0.001$.

Specifically, respondents reported higher health satisfaction $(M=6.12)$ when the study was intro- duced as a survey of Parkinson's patients rather than as a general population survey $(M=5.31)$, although this effect was not statistically significant; $F(1,96)=3.16, p<0.08$, Cohen's $d=0.36$ for the simple main effect.

In contrast, the life-satisfaction reports show the pattern of a part-whole contrast: respondents reported lower general life-satisfaction under the Parkinson's introduction $(\mathrm{M}=6.58)$ than under the general population introduction $(\mathrm{M}=7.44)$, $F(1,96)=4.19, p<0.05$, Cohen's $d=0.42$, for the simple main effect. This is consistent with a conversational process geared towards avoiding redundancy: Having just reported on their health satisfaction, at least some of the respondents disregarded this aspect of their lives when answering the general life-satisfaction question, as observed in previous studies $[4,8]$. See Table 2.

\section{Discussion}

In sum, these findings indicate that a preceding question about health satisfaction (Study 2) and an introduction that directs attention to health issues (Study 1) are functionally equivalent: Both result in increased correlations between health satisfaction and general life-satisfaction. Moreover, combining both manipulations does not further increase the observed accessibility effects.

\section{Conclusions}

Since the beginning of attitude research, researchers worried about the emergence of question order effects (for early discussions see Refs. $[16,17])$. In contrast, discussions of appropriate study introductions are mostly focused on ways in which the introduction could motivate respondents to participate in the study (e.g. [18]) and, more recently, on issues of informed consent (e.g. for a discussion see Ref. [19]). Both lines of thinking would suggest that it is a good idea to tell Parkinson's patients that the study in which they are asked to participate is concerned with Parkinson's patients and conducted by a university medical center in their area of residence. This information would increase the odds that respondents consider the study relevant to their own concerns and hence would presumably increase 
their motivation to participate. Moreover, this introduction would provide information that respondents need for truly informed consent, thus satisfying the requirements of Institutional Review Boards.

Unfortunately, this thinking captures only part of what is going on. As our findings illustrate, the information provided in introductions influences respondents' answers in much the same way as preceding questions do. Telling respondents that the study is concerned with the well-being of Parkinson's patients brings health-related information to mind, resulting in effects that mirror the nature of question order effects.

First, informing respondents that the study was concerned with the well-being of Parkinson's patients resulted in an inflated correlation between health and overall life-satisfaction relative to an introduction that did not mention health issues (Study 1). This effect parallels the influence of question order (e.g., [4, 6, 8]) and was observed even though the general life-satisfaction question preceded the health satisfaction question. In the present data, we would conclude that respondents' illness is a major determinant of their overall lifesatisfaction when the introduction brought their disease to mind, with health satisfaction accounting for $39.7 \%$ of the variance in reported life-satisfaction. Yet without a health-focused introduction, respondents' health satisfaction accounted for a more modest $11.5 \%$ of the variance in reported life-satisfaction. The latter estimate is roughly in line with a meta-analysis of studies of the general public, which suggests that selfreported health accounts for $9-14 \%$ of the variance in life-satisfaction [20]. These findings reiterate the lessons of question order experiments: Everything gets more important once it is brought to mind.

Second, study introductions may influence the nature of subsequent question order effects. In Study 2 we observed that respondents who had just reported their health satisfaction disregarded this information in a subsequent judgment of general life-satisfaction when the introduction framed the study as Parkinson's survey, but not when it framed the study as a general population survey. We surmise that the close relationship between the introduction, including the establishment of the respondent's diagnosis as part of the common ground, and the content of the first question sensitized respondents to potential redundancy of the question sequence. If so, study introductions may determine if a specific question results in assimilation effects or in subtractionbased contrast effects on a subsequent general question [9]. Study 2 suggests that this is the case, but more research is needed to bolster this conclusion.

Third, we found some suggestive evidence that introductions affect the frame of reference that respondents use in forming relevant judgments. Introducing a survey as a study of Parkinson's patients acknowledges the respondent's diagnosis as part of the common ground of the conversation between the researcher and the respondent $[10,12]$ and may increase the likelihood that respondents use other Parkinson's patients as the relevant comparison group. Once respondents evaluate their health relative to other patients, their judgments then are more positive than would otherwise be the case (Study 2). Our data suggest that this effect is limited to questions that pertain to the defining group characteristic, like health judgments in the present study. Specifically, we observed a marginally significant shift in reference standards for health satisfaction (Study 2), but not for general life-satisfaction (Study 1). However, we should note that the effect in Study 2 was of small to moderate size (Cohen's $d=0.36$ ), and was not statistically significant by conventional standards. Additional research is needed to determine if this effect might be more robust than was observed here, and to understand the conditions under which frame of reference shifts will or will not occur.

Our studies are limited in that they both focus on a single patient group, and rely on a similar methodology - brief phone surveys. In addition, because our items of interest were asked directly after the study introduction, we cannot say for sure how long the context effect would endure. In general, question order effects decrease when related questions are separated by several filler items (for a review see Ref. [21]) and this attenuation is more pronounced for older than for younger respondents, due to age-related declines in memory [22]. This means that placing several innocuous filler questions before the focal items of interest may decrease the biasing effects of the 
introduction. On the other hand, introductions identify the purpose and goal of the ongoing conversation and this information may be less likely to fade from memory than the content of a specific question. In addition, many surveys introduce information about the purpose of the study several times, for example, in an advance letter, in written consent process, and in the oral introduction, thus making it more memorable than was the case in our studies. Further studies would be needed to illuminate these issues. Finally, the gender composition of our two studies differed. Although this is not a threat to the internal validity of either study, this difference may limit our ability to directly compare the results of the two studies to one another.

Irrespective of these open questions, our findings converge on an important methodological conclusion: Study introductions can profoundly affect the answers respondents provide. The underlying processes can be fruitfully conceptualized in terms of the cognitive and communicative processes that drive the emergence of question order effects [3, 9]. As a result, researchers face a difficult trade-off. On the one hand, information that renders the study relevant to respondents' personal lives is likely to increase the response rate. Moreover, full disclosure of the purpose of the study enables respondents to provide fully informed consent. On the other hand, that same information is likely to distort the obtained results by bringing information to mind that respondents may otherwise not consider.

It may often be the case that in order to reduce bias, keeping study introductions as uninformative as possible may be desirable. Do we propose not to obtain informed consent? No. As our procedures illustrate, fully informed consent can be obtained at the end of the interview, when respondents can be informed about the purpose of the study and are fully aware of the information they have disclosed. This has been a standard approach in certain kinds of behavioral research that would be impossible to conduct if participants were aware of the study hypotheses prior to participation. Nonetheless, this approach may have considerable costs if it reduces trust between research participants and investigators. In our studies, only four respondents declined to allow us to use their data, and no respondents complained about our proce- dures. Nonetheless, it is certainly possible that some were upset and did not tell us, or that all of these participants may be more likely to be suspicious of researchers in the future. Ultimately, our data do not speak to how the tradeoff between fully informed prior consent and the risk of substantial bias should be resolved; this will have to be addressed on a case by case basis.

Finally, our findings highlight that patients' health plays a more prominent role in judgments of life-satisfaction when their attention is drawn to their illness than when it is not, be it through the introduction (Study 1) or through preceding questions (Study 2). Although this finding is consistent with previous research into the context dependency of judgments of well-being (for a review see Ref. [5]), many readers may wonder why a serious illness like Parkinson's disease isn't always on patients' minds. We suggest a simple answer: Nobody is a patient for 24 hours a day, just like students do not continuously obsess about dating [4] and spouses do not continuously obsess about their marriages [8]. The answers we collect in health-focused studies capture how patients feel when their illness is on their mind. Yet their illness may not be on their mind for most of the day. One methodological response to this dilemma is the development of duration-weighted measures that assess respondents' subjective well-being in different contexts and weight the responses by the time the person spends in the respective situations (for an example see Ref. [23]); another is the use of experience sampling methods (e.g., [24]). Without such precautions, we run the risk that our conclusions about the relative importance of different aspects of life are driven by the focusing effects induced by our research instrument.

\section{Notes}

1. Both life and health satisfaction were measured as ordinal, ten-point scales. However, in our analyses we treated them as continuous variables, reflecting the theoretical position that the underlying satisfaction constructs are continuous. Although treating ordinal scales as continuous technically violates statistical assumptions, it is not considered problematic to do so when a sufficient number of response categories are 
used (e.g., at least five). In a review of the literature, Jaccard and Wan [25] conclude that using ordinal scales in linear analyses does not result in substantial problems with Type I or Type II error, even in cases where doing so results in large departures from intervalness.

2. The rest of the regression model is as follows: Regression coefficient for condition $=2.16$ $(0.79)$, for health satisfaction $=0.58(0.09)$, intercept $=3.33(0.59)$.

\section{Acknowledgements}

This research was supported by the National Institute of Child Health and Human Development (R01HD040789). Dylan Smith is supported by a career development award from the VA. Peter Ubel receives funding from a Presidential Early Career Award for Scientists and Engineers.

\section{References}

1. Schwarz N. Self-reports: How the questions shape the answers. Am Psychol 1999; 54: 93-105.

2. Tourangeau R, Rips LJ, Rasinski K. The Psychology of Survey Response. Cambridge, UK: Cambridge University Press, 2000

3. Sudman S, Bradburn N, Schwarz N. Thinking About Answers: The Application of Cognitive Processes to Survey Methodology. San Francisco, CA: Jossey-Bass, 1996.

4. Strack F, Martin LL, Schwarz N. Priming and communication: The social determinants of information use in judgments of life-satisfaction. Eur J Soc Psychol 1988; 18: 429-442.

5. Schwarz N, Strack F. Reports of subjective well-being: Judgmental processes and their methodological implications. In: Kahneman D, Diener E, Schwarz N (eds.), Well-being: The Foundations of Hedonic Psychology. New York: Russell-Sage, 1999: 61-84.

6. Haberstroh S, Oyserman D, Schwarz N, Kühnen U, Ji LJ. Is the interdependent self more sensitive to question context than the independent self? Self-construal and the observation of conversational norms. J Exp Soc Psychol 2002; 38: 323-329.

7. Oishi S, Schimmack U, Colcombe SJ. The contextual and systematic nature of life-satisfaction judgments. J Exp Soc Psychol 2003; 39: 232-247.

8. Schwarz N, Strack F, Mai HP. Assimilation and contrast effects in part-whole question sequences: A conversational logic analysis. Public Opin Q 1991; 55: 3-23.

9. Schwarz N, Bless H. Constructing reality and its alternatives: Assimilation and contrast effects in social judgment. In: Martin LL, Tesser A (eds.), The Construction of Social Judgment. Hillsdale, NJ: Erlbaum, 1992: 217-245.
10. Schwarz N. Cognition and Communication: Judgmental Biases, Research Methods and the Logic of Conversation. Hillsdale, NJ: Erlbaum, 1996.

11. Norenzayan A, Schwarz N. Telling what they want to know: Participants tailor causal attributions to researchers' interests. Eur J Soc Psychol 1999; 29: 1011-1020.

12. Clark HH, Schober MF. Asking questions and influencing answers. In: Tanur JM (ed.), Questions About Questions. New York: Russel Sage, 1992: 15-48.

13. Schuman H, Presser S. Questions and Answers in Attitude Surveys. New York: Academic Press, 1981.

14. Grice HP. Logic and conversation. In: Cole P, Morgan JL (eds.), Syntax and Semantics, Vol.3: Speech Acts. New York: Academic Press, 1975: 41-58.

15. Clark HH, Haviland SE. Comprehension and the givennew contract. In: Freedle RO (ed.), Discourse Production and Comprehension. Hillsdale, NJ: Erlbaum, 1977: 1-40.

16. Cantril H. Gauging Public Opinion. Princeton, NJ: Princeton University Press, 1944.

17. Payne SL. The Art of Asking Questions. Princeton: Princeton University Press, 1951.

18. Groves RM, Cialdini RB, Couper MP. Understanding the decision to participate in a survey. Public Opin Q 1992; 56: 475-495.

19. Groves RM, Fowler FJ, Couper MP, Lepkowski JM, Singer E, Tourangeau R. Survey Methodology. New York: Wiley, 2004.

20. Okun MA, Stock WA, Haring MJ, Witter RA. Health and subjective well-being: A Meta-Analysis. Int J Aging Hum Dev 1984; 19: 111-132.

21. Wänke M, Schwarz N. Reducing question order effects: The operation of buffer items. In: Lyberg L, Biemer P, Collins M, DeLeeuw E, Dippo C, Schwarz N (eds.), Survey Measurement and Process Quality. Chichester, UK: Wiley, 1997: 115-140.

22. Schwarz N, Knäuper B. Cognition, aging, and self-reports. In: Park D, Schwarz N (eds.), Cognitive Aging. A primer. Philadelphia, PA: Psychology Press, 2000: 233-252.

23. Kahneman D, Krueger AB, Schkade D, Schwarz N, Stone AA. A survey method for characterizing daily life experience: The Day Reconstruction Method (DRM). Science 2004; 306: 1776-1780.

24. Riis J, Loewenstein G, Baron J, Jepson C, Fagerlin A, Ubel PA. Ignorance of hedonic adaptation to Hemo-Dialysis: A study using ecological momentary assessment. J Exp Psychol Gen 2005; 134(1): 3-9.

25. Jaccard J, Wan CK. LISREL Approaches to Interaction Effects in Multiple Regression. Thousand Oaks: Sage Publications, 1996.

26. Frederick S, Loewenstein G. Hedonic adaptation. In: Kahneman D, Diener E, Schwarz N (eds.), Well-being: Foundations of Hedonic Psychology. New York: RussellSage, 1999: 302-329.

Address for correspondence: Dylan M. Smith, 300 N. Ingalls Rm. \# 7D19, University of Michigan, Ann Arbor, MI 481090429, USA

Fax: + 1-734-936-8944

E-mail: dylsmith@umich.edu 